\title{
Tracking Development of the Corpus Callosum in Fetal and Early Postnatal Baboons Using Magnetic Resonance Imaging
}

\author{
Kimberley A. Phillips ${ }^{1,2,3}$ and Peter Kochunov*,2,3
}

\author{
${ }^{1}$ Department of Psychology, Trinity University, San Antonio Texas, USA; ${ }^{2}$ Research Imaging Institute, University of \\ Texas Health Science Center at San Antonio, Texas, USA; ${ }^{3}$ Southwest Foundation for Biomedical Research, San Anto- \\ nio, Texas, USA
}

\begin{abstract}
Although the maturation of the corpus callosum (CC) can serve as a sensitive marker for normative antenatal and postnatal brain development, little is known about its development across this critical period. While high-resolution magnetic resonance imaging can provide an opportunity to examine normative brain development in humans, concerns remain over the exposure of developing fetuses to non-essential imaging. Nonhuman primates can provide a valuable model for normative brain maturation. Baboons share several important developmental characteristics with humans, including a highly orchestrated pattern of cerebral development. Developmental changes in total CC area and its subdivisions were examined across the antenatal (weeks 17 - 26 of 28 weeks total gestation) and early postnatal (to week 32) period in baboons (Papio hamadryas anubis). Thirteen fetal and sixteen infant baboons were studied using high-resolution MRI. During the period of primary gyrification, the total area of the CC increased by a magnitude of five. By postnatal week 32, the total CC area attained only $51 \%$ of the average adult area. CC subdivisions showed non-uniform increases in area, throughout development. The splenium showed the most maturation by postnatal week 32 , attaining $55 \%$ of the average adult value. The subdivisions of the genu and anterior midbody showed the least maturation by postnatal week 32 , attaining $50 \%$ and $49 \%$ of the average adult area. Thus, the CC of baboons shows continued growth past the postnatal period. These age-related changes in the developing baboon CC are consistent with the developmental course in humans.
\end{abstract}

Keywords: Brain development, In-utero, Fetal MRI, Neurogenesis.

\section{INTRODUCTION}

Cerebral development in primates during the fetal and early postnatal period involves coordinated biochemical and structural changes including histogenesis (including neurogenesis and gliogenesis), migration, and myelination. Understanding cerebral development in utero is especially important, as abnormal development during this period is believed to contribute to the etiology of many neurodevelopmental disorders, including autism spectrum disorder, attention deficit disorder and mood disorders [1-4].

The corpus callosum (CC) is the largest commissural white matter tract in placental mammalian brains, connecting both homotopic and heterotopic regions of the cerebral cortex [5]. The CC is part of the latest maturing networks of the brain, and is essential for the interhemispheric integration of sensory, motor and higher-order cognitive information. Thus, its development and maturation serves as a sensitive indicator of normal brain development. However, while prenatal abnormalities of the CC have been reported [6, 7], little is known about its normal development from in utero to maturation.

\footnotetext{
*Address correspondence to this author at the University of Texas Health Science Center at San Antonio, Research Imaging Institute Maryland Psychiatric Research Center, Department of Psychiatry, University of Maryland, School of Medicine, 55 Wade Avenue, Catonsville, MD 21228;

Tel: (410) 402-6110; Fax: (410) 402-6077;

E-mail: pkochunov@mprc.umaryland.edu
}

In the human embryo, callosal axons appear around 74 days $[8,9]$. The subdivisions of the genu and splenium can be identified at 84 days and the adult morphology is recognized by 115 days [8]. Measurements of callosal width, length and thickness from magnetic resonance [10] imaging are possible from gestational week 16 [11]. Over the gestational course, the thickness and width of the CC increase by a magnitude of three [11]. During the first few postnatal months, a growth spurt is seen in the genu of the CC, followed by a period of rapid growth in the splenium [12]. By eight months, the CC has an adult appearance on a midsagittal MR image. The CC continues to mature throughout childhood and adolescence. Ongoing regressive and progressive maturational events result in a functional reorganization of the CC [13].

While investigation of fetal and early postnatal brain development can be accomplished using high-resolution magnetic resonance imaging (MRI), concerns remain over the exposure of developing fetuses to nonessential imaging [14]. Thus, nonhuman primates (NHP) can serve as a valuable model for human cerebral development. Of NHP, no species other than apes are closer to humans from an evolutionary perspective than are baboons (Papio spp.) [15]. Baboons share several important characteristics of development with humans, including a prolonged infancy and juvenile period, long lifespan, and complex social behavior; they are ideal models for investigating cerebral development and are likely to further our understanding of neurodevelopmental disorders, psychiatric conditions and disease [2]. 
Baboons share a highly orchestrated pattern of cerebral development with humans. Formation of the cerebral landscape, or primary gyrification (PG), begins in the early stages of telencephalic development. Its onset coincides with completion of neuronal proliferation and migration; its progression is accompanied by an explosive increase in cerebral growth $[10,16,17]$. The onset of PG is coupled with the beginning of neuronal differentiation that partitions the cortical lamina into an intricate mosaic of specialized functional areas [18]. Primary cortical sulci and gyri appear in a tightly controlled ontogenic sequence during PG; this pattern is highly conserved across primates, including humans $[10,17$, 19]. The baboon CC can be visualized at 110 days gestation [20]; however, the relation of CC growth to PG is unclear.

Given the importance of the CC to fundamental human cognitive abilities and as a marker of normal brain development, it is important to understand the development of this structure in a primate that displays similarity to humans in other aspects of neuroanatomical maturation. In this report, we used high-resolution MRI to quantify the development of CC in fetal and early postnatal baboons. We relate the growth of the CC to PG in baboons to more fully understand the integrated development of these two key components of cerebral development.

The main challenge in imaging of NHP is that adult brain volumes are much smaller than the human brain volume by a factor of 2-200. This difference in size is further accentuated in NHP studies of antenatal brain development in which fetal brain volumes are $20-50 \%$ of their adult size. An important consideration in the design of an imaging protocol is spatial resolution with which brain morphology is sampled. While the brain volumes of NHP are much smaller than human brains, not all internal brain structures scale allometrically with brain volume. For example, the average thickness of cerebral cortex is very similar among all mammals (about 2 $\mathrm{mm}$ ) and yet the characteristic sizes of the morphological features of cortical landscape structure, gyri and sulci, scale directly with brain volume [21]. We designed an imaging protocol for allometric spatial sampling in baboon brain [22]. Our protocol uses an isotropic spatial sampling volume of 150 microns $^{3}$ (isotropic 500 micron resolution). This provides the spatial sampling of the gyral and sulcal structures comparable to high resolution high resolution human studies $\left(\sim 1.0 \mathrm{~mm}^{3}\right)$ in a primate whose brain volume is $1 / 8$ the volume of a human brain. Likewise, imaging at this spatial resolution yields a much finer sampling of the cortical gray matter ribbon whose thickness is similar in baboons and humans, while providing sufficient signal-to-noise ratio for semiautomated and automated imaging process [22].

\section{METHOD}

\section{Pregnant Animals}

A breeding colony of olive baboons (Papio hamadryas anubis) has been maintained for more than 40 years by the Southwest National Primate Research Center (SNPRC) at the Southwest Foundation for Biomedical Research in San Antonio, Texas. In utero imaging of 13 normally developing fetuses was performed covering the period of gestational week 17 through birth (gestational week 28); imaging was performed on an additional 16 baboons up to postnatal week
32. Fetal gestation age was calculated from the date of birth [23].

\section{Handling and Anesthesia}

Animals were transported from the SNPRC to the Research Imaging Institute, University of Texas Health Sciences Center at San Antonio for imaging. Handling and anesthesia procedures followed previously described procedures [24]. In short, 15 minutes prior to scanning, animals were immobilized with ketamine $(10 \mathrm{mg} / \mathrm{kg})$ and intubated with a MR-compatible endotracheal tube. Anesthesia was maintained with $5 \%$ isoflurane with an MR-compatible gas anesthesia machine. Animals remained anaesthetized throughout the MRI procedure and respiration rate, heart rate, and oxygen consumption were continually monitored. This protocol and all animal procedures were reviewed and approved by the Institutional Animal Care and Use Committee of the Southwest Foundation for Biomedical Research.

\section{Image Acquisition}

The methodological details of the in utero and post natal imaging are discussed elsewhere [22]. High-resolution (isotropic $500 \mu \mathrm{m}$ ) images of the fetal baboon brain were acquired with a 3D, balanced steady-state free precession pulse protocol with respiratory gating and retrospective motion correction (Kochunov and Davis 2009). The sequence control parameters TR/TE/flip angle/FOV/Spatial Resolution $/$ Scan Time $=5 \mathrm{~ms} / 2.5 \mathrm{~ms} / 75$ degrees $/ 180 \mathrm{~mm} / 500$ microns isotropic/30 min, allow for rapid collection of 3D data phase partition of 360 lines within a single respiration cycle. Our objectives in development of this protocol were to achieve high-SNR and 3D and isotropic coverage of a fetal brain with good, regional gray matter - white matter tissue contrast (Fig. 1, top). The choice of sequences for in utero imaging is generally limited to sequences that emphasize tissue differences in T2 relaxation times (T2-weighted). In the unmyelinated fetal brain, there is little T1-weighted GM/WM contrast and additional complications arise due to amniotic fluid that surrounds the fetal head. Its T1-relaxation time is similar to that of brain tissue, thus complicating segmentation procedures. Fortunately, there is sufficient difference in the T2 relaxation times among cerebral WM, GM and amniotic fluid ( 2000 ms), allowing for sufficient brain tissue contrast and excellent brain/amniotic fluid contrast [22]. We chose a 3D balanced gradient echo (TrueFisp) sequence as this sequence provides the highest level of signal per TRinterval among all MR sequences. While the image contrast in a TrueFisp pulse sequence depends on the T2/T1 ratio as opposed to T2, in fetal brain imaging, the tissue contrast is qualitatively similar to that of the T2-weithed sequences, providing an excellent regional contrast in fetal cerebrum (Fig. 2).

Two additional factors that make fetal brain imaging technically challenging are the periodic, respiratory motion of the mother's diaphragm and fetal head movement [22, 25]. To suppress artifacts association with respiratory motion we used respiratory gating in combination with mechanical ventilation. During respiratory gating, the animal breathing pattern is monitored using the tidal volume measurement provided by the mechanical ventilator. When the diaphragm is fully expanded, a trigger signal is sent to the host computer. Following a trigger delay time, set to be sufficiently 


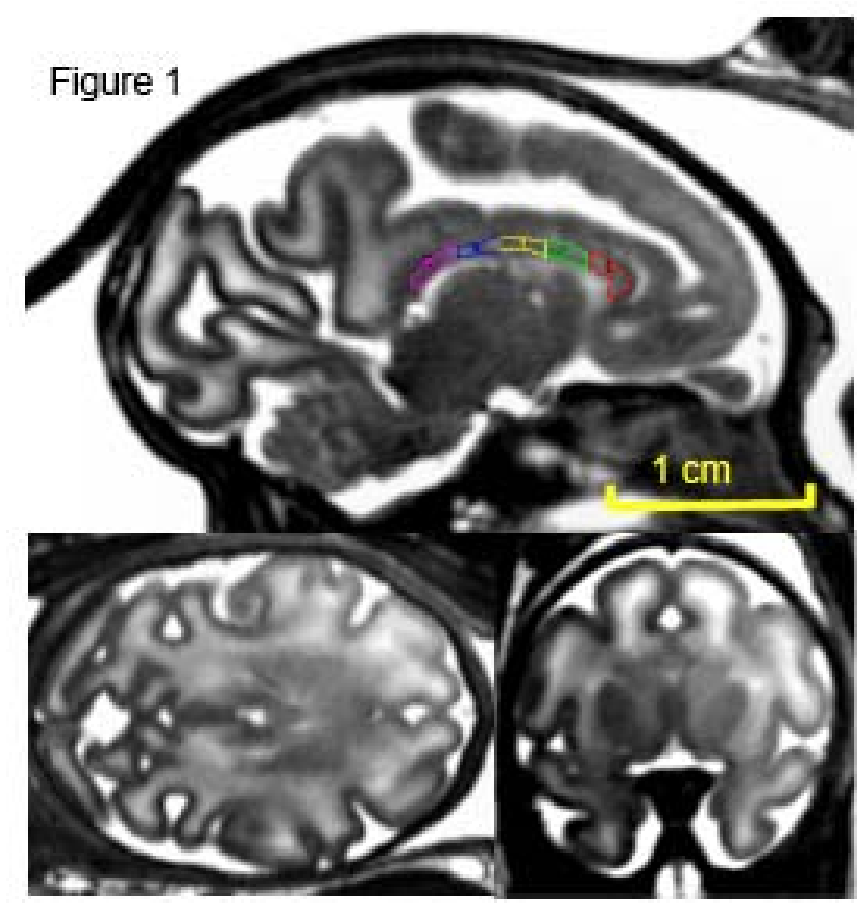

-8 weeks

Fig. (1). Mid-sagittal (top) axial (left) and coronal (right) slices of a fetus at week 20 (of 28-week long term) of in utero development. The high-resolution (isotropic 500 $\mu \mathrm{m}$ ) SSFP images show good ( 20\%) GM/WM contrast and signal to noise ration ( 30) that allows for delineation of corpus callosum. The corpus callosum is traced, and subdivided into five regions, on the midsagittal section at each age. $1=$ genu, 2 = anterior midbody, 3 = medial midbody, 4 = caudal midbody, and 5 = splenium.

long for an animal to exhale, data acquisition is performed in the 3-5 second intervals when the diaphragm is at rest. To correct for potential fetal head movement, we acquired structural images using a retrospective motion-corrected approach [26]. High-resolution spatial sampling and the need for sufficient SNR translate into a long scan time of 20-30 min and this inevitably leads to the increased likelihood of motion even in anesthetized animals. To prevent motionrelated artifacts we partitioned motion into two categories: intra- and inter-scan. Intra-scan motion leads to blurring and phase-related artifacts. Such artifacts cannot be adequately corrected. In contrast, inter-scan movements can be modeled as a 3-D rigid-body motion and corrected using spatial realignment. To reduce the intra-scan fetal head motion, we segmented image acquisition into intervals of only 2-3 min long. The time-segmentation of data acquisitions helped us to cast the intra-scan motion into the inter-scan category which can be modeled and corrected [26].

A 3D, magnetization-prepared, transient steady state, rapid gradient echo (MPRAGE) sequence with isotropic 500 $\mu \mathrm{m}$ resolution was chosen to study post-natal development [22]. This sequence provides an excellent T1-weighted contrast that is uniform across the brain volume and can be used to differentiate between gray matter and myelinated and unmyelinated white matter [27]. The sequence control parameters $\left(\mathrm{FOV}=128 \mathrm{~mm}, \mathrm{TI}=795 \mathrm{~ms}, \mathrm{TE}=3.04, \mathrm{TR}_{1}=5 \mathrm{~ms}\right.$, $\mathrm{TR}_{2}=2000 \mathrm{~ms}$ and flip angle $=10$ degrees) were modeled to produce GM/WM contrast of $25 \%$ based on the analytical solutions to Bloch equations [28] and average measured values of T1, T2 and PD. Image acquisition was performed using a retrospective motion-corrected protocol [29]. Under this protocol, six full resolution segments, 9 min. long each, were acquired for a total sequence running time of $\sim 54 \mathrm{~min}$.

\section{Image Quantification}

Measures of CC area were taken from T2-weighted TrueFisp images, which allow for better differentiation of cortex and white matter in fetal and postnatal brains. Prior to morphometric analysis, data were converted into the ANALYZE 3D volume file format to facilitate re-slicing into orthogonal planes. 3D volumes for individual subjects were numerically coded prior to measurement to prevent observer bias. Each individual brain was realigned along the AC-PC and interhemispheric fissure. CC area measurements were performed using manual tracings on the midsagittal slice according to the methodology that was originally described by Biegon and colleagues [30] and later adapted to NHP by Sanchez et al. [31]. In the original procedure, the anterior $20 \%$ of the CC was defined as the genu, the posterior $20 \%$ defined as splenium, and the middle $60 \%$ defined as body. To adapt this to NHP studies, Sanchez et al. [31], Phillips et al. [32], and Pierre et al. [33] further delineated the middle $60 \%$ into three equal regions: anterior midbody, medial midbody, and caudal midbody. These subdivisions of the CC are believed to be based on microstructure and functional connectivity with cortical areas [34-36]. The anterior region of the genu connects primarily higher-order cognitive regions; the anterior, medial and caudal midbody connect primarily sensorimotor regions; the posterior region of the splenium integrates visuospatial regions of the cortex. ANALYZE 10.0, an MRI analysis software program distributed by the Mayo Clinic, was used to divide and measure the CC 


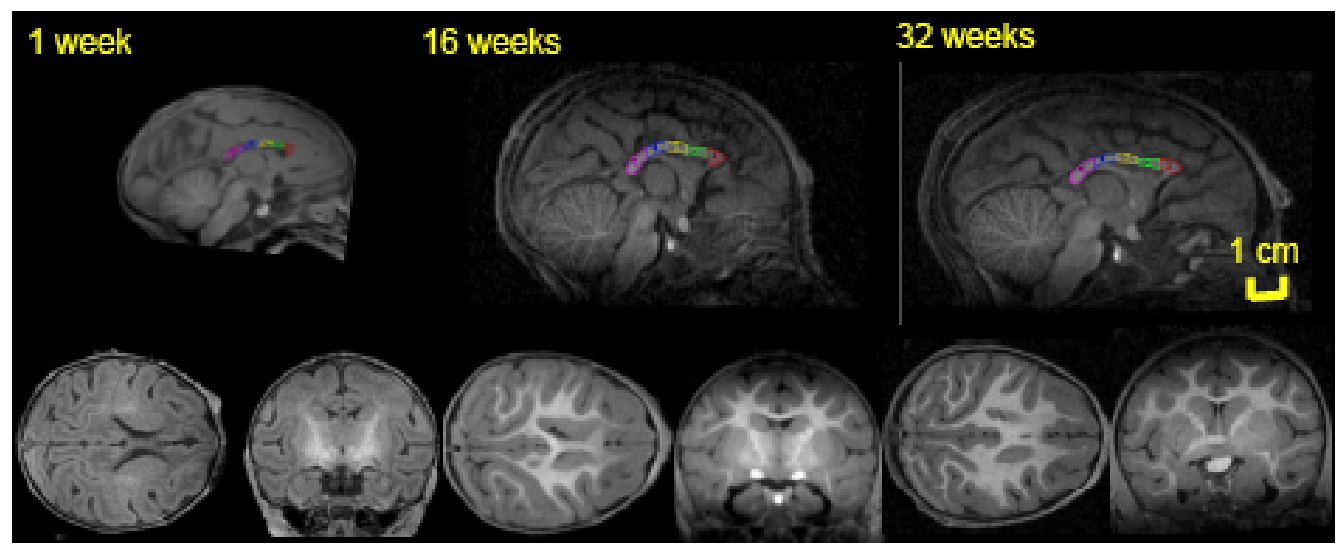

Fig. (2). Mid-sagittal (top) axial (left) and coronal (right) slices of baboon brain at week 1 (left), 16 (middle) and 32 (right) of postnatal de-

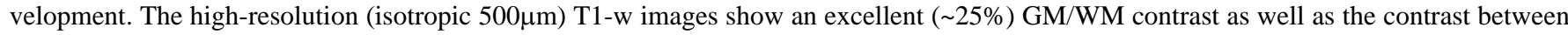
myelinated and unmyelinated WM. The corpus callosum is traced, and subdivided into five regions, on the midsagittal section at each age. 1 = genu, 2 = anterior midbody, 3 = medial midbody, 4 = caudal midbody, and $5=$ splenium.

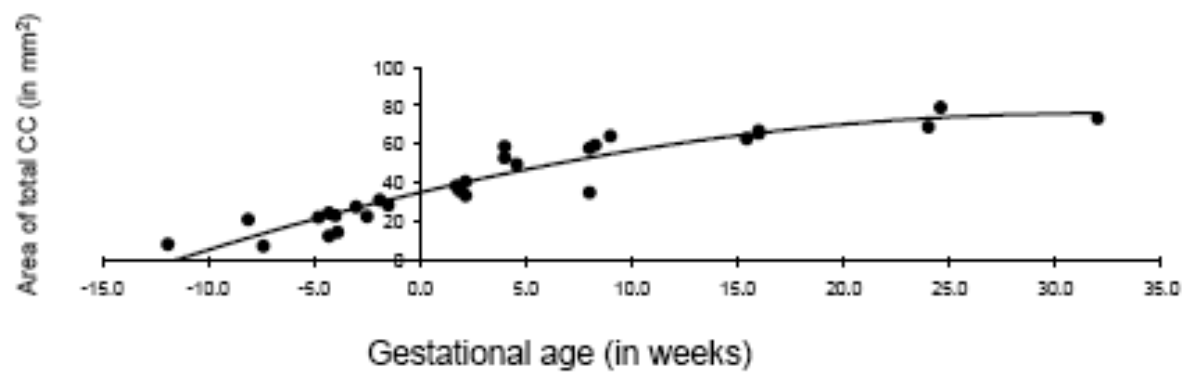

Fig. (3). Age-related change in the total corpus callosum in fetal and early postnatal baboons is best described by a quadratic curve (area $=$ $.041 *$ age $^{2}+2.59 *$ age $\left.+34.72 ; R^{2}=.89\right)$.

(in $\mathrm{mm}^{2}$ ). To subdivide the CC, the entire length of the CC was first manually traced, and then divided into five equally spaced sections.

Statistical analysis. Changes in the total CC and CC subdivision areas were analyzed using ANCOVA to calculate the significance of sex and age effects. These analyses were conducted using a generalized linear model that included linear and quadratic effects of age and linear effects of sex and age. Both linear and quadratic terms were included, given previous reports of developmental patterns in the human CC $[37,38]$. This approach was used to avoid the problem of multicollinearity on the total CC area (as total CC is a linear combination of the five CC subdivisions). Sex was used a fixed factor because testosterone modulates regional callosal architecture producing gender-specific differences in regional development [39]. F tests were used to determine whether linear or quadratic growth models best fit the developmental change in these regions [40-44].

\section{RESULTS}

At gestational week 17 the CC could be readily identified, the total area of the CC in this subject measured 7.68 $\mathrm{mm}^{2}$. By postnatal week 32, the total area of the CC has increased to $73.47 \mathrm{~mm}^{2}$. Total CC area did not differ significantly by $\operatorname{sex}(F(1,26)=2.88, p=.102)$. Total CC area was significantly affected by the covariate age $(F(1,26)=$ 145.23, $p<.001)$. All subdivisions of CC were significantly affected by the covariate age [genu: $F(1,26)=66.16, p<$ .001 ; anterior midbody: $F(1,26)=73.76, p<.001$; medial midbody: $F(1,26)=86.70, p<.001$; caudal midbody: $F(1$, $26)=155.19, p<.001$; splenium: $F(1,26)=116.36, p<$ $.001)$. The subdivision of the anterior midbody was significantly influenced by sex, with females having larger raw areas than males (anterior midbody: female $M=8.38$, $S D=$ 4.48; male $M=6.22, S D=3.61 ; F(1,26)=13.05, p=.001)$. Sex differences were not detected for any other CC subdivision.

The total area of the CC displayed significant age-related change across the fetal and early postnatal period and was best explained by quadratic growth $(F(2,26)=108.99, p<$ $0.001 ; R^{2}=.89$; see Fig. 3 ). Changes in the genu and splenium were similarly explained by linear and quadratic growth [genu: linear: $F(1,27)=65.23, p<0.001 ; R^{2}=.70$; quadratic: $F(2,26)=37.84, p<0.001 ; R^{2}=.73$; splenium: linear: $F(1,27)=117.39, p<0.001 ; R^{2}=.81$; quadratic: $F$ $\left.(2,26)=76.02, p<0.001 ; R^{2}=.84\right]$. Growth in the anterior midbody, medial midbody and caudal midbody was best explained by quadratic growth [anterior midbody: $F(2,26)=$ 31.75, $p<0.001 ; R^{2}=.69$; medial midbody: $F(2,26)=$ 68.93, $p<0.001 ; R^{2}=.83$; caudal midbody: $F(2,26)=$ 163.35, $\left.p<0.001 ; R^{2}=.92\right]$. Growth curves for all subdivisions of the CC are displayed in Figs. (4a-e).

\section{DISCUSSION}

To our knowledge, this is the first study to analyze changes in CC area during primary gyrification and early postnatal development. Primary gyrification (PG) in baboons occurs rapidly over a nine week period in the early stages of 


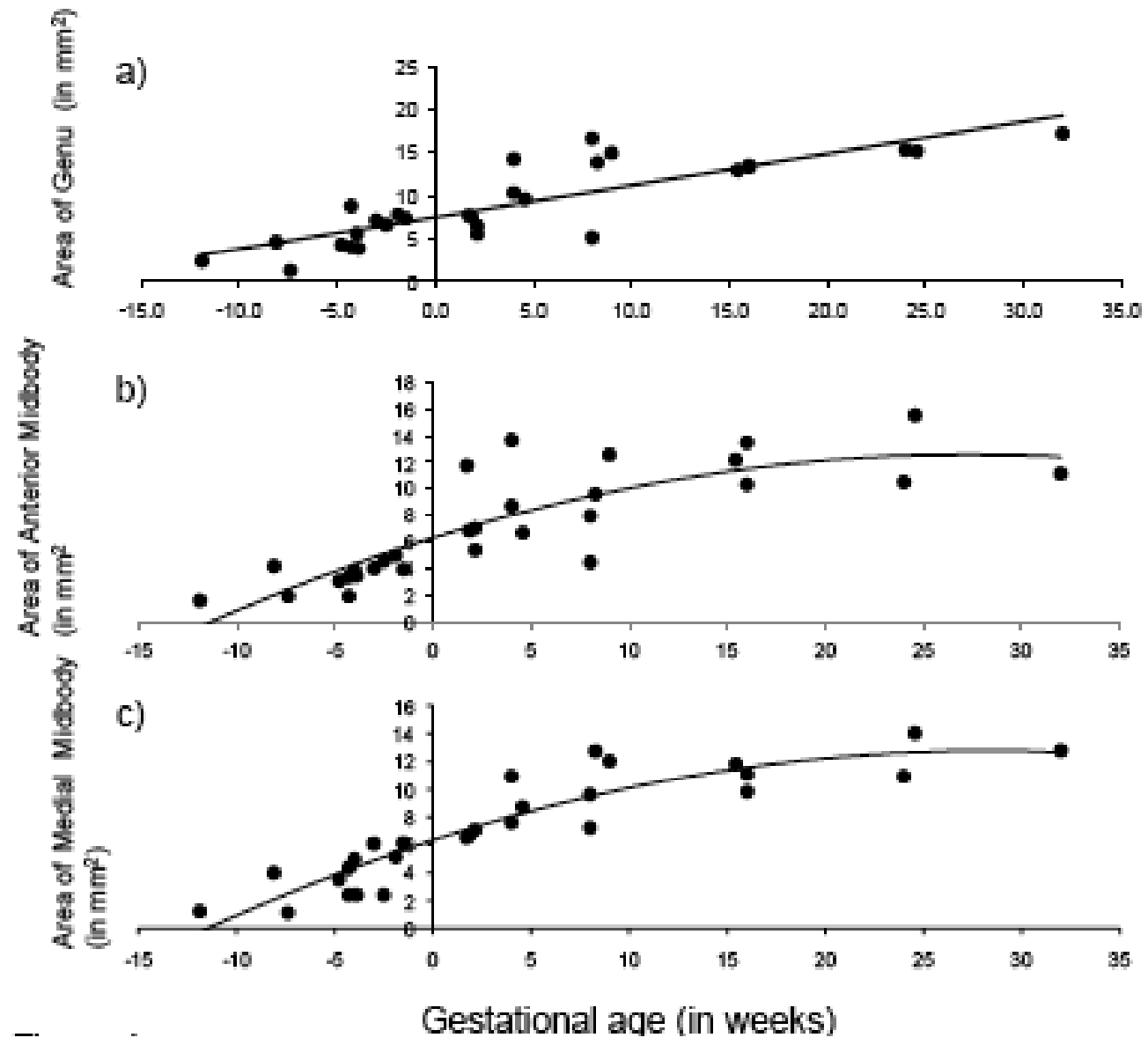

Fig. (4). Age-related change in CC subdivisions in fetal and early postnatal baboons: A) genu (area $=.368 *$ age $+7.52 ; R^{2}=.71$ ), B) anterior midbody (area $=-.008 *$ age $^{2}+.462 *$ age $\left.+6.29 ; R^{2}=.69\right)$, C) medial midbody (area $=-.008 *$ age $^{2}+.457 *$ age $+6.31 ; R^{2}=.83$ ), D) caudal midbody (area $=-.008 *$ age $^{2}+.495 *$ age $\left.+5.91 ; R^{2}=.92\right)$, and E) splenium (area = -.009*age ${ }^{2}+.684 *$ age $\left.+8.36 ; R^{2}=.84\right)$.

telencephalic development, from gestational week 17 to gestational week 26 [25]. During PG, the cross-sectional area of CC in fetal baboons increased by a magnitude of five. The rapid growth of CC continued past partition, doubling in size by week 32 of postnatal development. At that time, the size of CC was only $51 \%$ of the average adult CC area (142.98 \pm $22.09 \mathrm{~mm}^{2}$ ) indicating that maturation of the baboon CC continues past the early postnatal period. The growth for different subdivisions of CC was not uniform. At week 32, the genu achieved $50 \%$ of the average adult value, the anterior midbody achieved $49 \%$, the medial midbody $53 \%$, and caudal midbody $48 \%$, and the splenium achieved $55 \%$ of the average adult value. Growth in the subdivisions of the genu and splenium were similarly explained by linear and quadratic equations, increasing in area across the fetal and early postnatal period. For the anterior midbody, medial midbody and caudal midbody regions, the quadratic equation accounted for a significantly greater amount of variability compared to the linear equation, indicating that these subdivisions displayed non-linear growth.

Developmental changes in cross-sectional area of CC in humans correspond to increased axonal myelination rather than changes in axonal density [34, 45]. The same mechanisms are presumed to be responsible for our findings. Based on developmental data of the CC in chimpanzees and capuchin monkeys indicating continued maturation into adult- hood [40, 46], we would expect the baboon CC to also display continued growth into adulthood.

While sex differences in the size of the baboon CC were not detected for total CC area, sex differences were found in anterior midbody. Several factors may explain why we did not detect sex differences in the development of the baboon CC. While the sample was balanced with respect to distribution of males and females in both the fetal and early postnatal period, the overall sample size was limited (13 fetal subjects, 16 postnatal subjects). Variability across samples may have masked potential sex differences in development. Additionally, it may be that during this early developmental stage there are no sex differences present in the baboon CC.

Whether or not sex differences are present in the development of the human CC across childhood and adolescence is controversial, as reports are inconsistent [37, 41, 47-50]. However, different methodologies, including varying sample sizes, ages, and means of quantifying the CC, may explain these discrepancies. In fact, when controlling for these variables, human males and females display significant differences in the maturation of the CC and CC subdivisions [51]. These sex differences vary as a function of callosal region and developmental stage. Postnatal structural changes in the human $\mathrm{CC}$ are likely due to myelination, pruning, and axonal redirection [52, 53]. 
In the present study, the CC was subdivided into five equal sections, following methodology of previous researchers [31-33]. These subdivisions are believed to be related to microstructure and functional connectivity based upon histological study [34]. It was not possible to collect histological data on this baboon sample to measure axon diameter distribution across the CC. Implementation of technologies such as diffusion tensor imaging (to track fiber projections) and AxCaliber (to measure axon diameter distribution in vivo) [54] would provide a refinement of callosal subdivisions and their growth trajectories.

Scientists are challenged to seek the most appropriate animal model of biological relevance to a given question and then to match that model with the measurement tool(s) best suited to test their hypothesis. Non-human primate (NHP) models for both normative and perturbed biology can yield invaluable findings, but NHP models are uniquely suitable for translational imaging research in genetics, morphology and maturation of cortical landscape [16, 55-57]. Genetically, Old World primates share up to $99 \%$ of the human genome [55]. The reduced complexity of the primate brain and compressed rate of cerebral development make NHPs a valuable research model to study brain maturation.

\section{CONCLUDING REMARKS}

Structural imaging research in NHPs is a rapidly growing field. MRI studies of NHPs can be used not only to validate basic neuroscience theories regarding development of cerebral cortex and CC, but also to accelerate the development of new drugs and medical devices. Several challenges must be overcome before achieving the same level of allometric sampling and tissue signals in NHPs as currently available in humans, due to their smaller brain volumes. Fortunately, emerging improvements in gradient and multi-channel RF electronics, acquisition software, along with higher magnetic field strengths allow for the acquisition of NHP imaging data that are compatible to the best structural data from humans (based on brain volumes). As presented here, careful optimization of MRI acquisition protocols and suppression of motion artifacts can produce highly reliable, quantitative data. Using these, or similar, strategies enables investigators to study NHP brain development using the wealth of computational tools already developed for humans.

\section{ACKNOWLEDGEMENTS}

We thank our collaborators for some of the material presented in this paper; Drs. Jeffery Rogers (Baylor Univ.), Steven Leigh (Univ. Illinois), Gerald Schatten and Carlos Castro (Univ. Pittsburgh). We also acknowledge technical assistance from the Dept. of Comparative Medicine at the Southwest National Primate Research Center (SNPRC). This work was supported, in part, by the National Institute of Neurological Disorders and Stroke (NIH R15 \#NS070717) to KAP, the National Institute of Biomedical Imaging and Bioengineering (K01 \#EB006395) grant to PK, the SNPRC (NIH \#P51-RR013986), and NIH (\#P01 HD047675 and R24 RR013632) grants to GS.

\section{REFERENCES}

[1] Casey BJ, Nigg JT, Durston S. New potential leads in the biology and treatment of attention deficit-hyperactivity disorder. Curr Opin Neurol 2007; 20: 119-24.
[2] Dawson G, Ashman SB, Carver LJ. The role of early experience in shaping behavioral and brain development and its implications for social policy. Dev Psychopathol 2000; 12: 695-712.

[3] Kalia M. Brain development: anatomy, connectivity, adaptive plasticity, and toxicity. Metabolism 2008; 57 (Suppl. 2): S2-S5.

[4] Rapoport JL, Addington A, Frangou S. The neurodevelopmental model of schizophrenia: what can very early onset cases tell us? Curr Psychiatr Rep 2005; 7: 81-2.

[5] Tomasch J. Size, distribution, and number of fibers in the corpus callosum. Anat Rec 1954; 119: 119-35.

[6] Comstock CH, Culp D, Gonzalez J, Boal DB. Agenesis of the corpus callosum in the fetus: its evolution and significance. $\mathrm{J}$ Ultrasound Med 1985; 4: 613-6.

[7] Pilu G, Sandri F, Perolo A, et al. Sonography of fetal agenesis of corpus callosum: a survey of 35 cases. Ultrasound Obstet Gynecol 1993; 3: 318-29.

[8] Loser JD, Alvord EC. Agenesis of the corpus callosum. Brain 1968; 91: 553-70.

[9] Prayer D, Kasprian G, Krampl E, et al. MRI of normal fetal brain development. Eur J Radiol 2006; 57: 199-216.

[10] Armstrong E, Schleicher A, Omran H, Curtis M, Zilles K. The ontogeny of human gyrification. Cereb Cortex 1995; 5(1): 56-63.

[11] Achiron R, Achiron A. Development of the human fetal corpus callosum: a high-resolution, cross-sectional sonographic study. Ultrasound Obstet Gynecol 2001; 18: 343-7.

[12] Barkovich AJ, Kjos BO. Normal postnatal development of the corpus callosum as demonstrated by MR imaging. AJNR Am J Neuroradiol 1988; 9(3): 487-91.

[13] Westerhausen R, Luders E, Specht K, et al. Structural and functional reorganization of the corpus callosum between the age of 6 and 8 years. Cereb Cortex 2011; 21(5): 1012-7.

[14] Buls N, Covens P, Nieboer K, et al. Dealing with pregnancy in radiology: a think line between science, social and regulatory aspects. JBR - BTR 2009; 92: 271-9.

[15] Stewart CB, Disotell TR. Primate evolution - in and out of Africa. Cur Biol 1998; 8: R582-R8.

[16] Armstrong E, Curtis M, Buxhoeveden DP, et al. Cortical gyrification in the rhesus monkey: a test of the mechanical folding hypothesis. Cereb Cortex 1991; 1(5): 426-32.

[17] Pillay P, Manger PR. Order-specific quantitative patterns of cortical gyrification. Eur J Neurosci 2007; 25(9): 2705-12.

[18] Neal J, Takahashi M, Silva M, Tiao G, Walsh CA, Sheen VL. Insights into the gyrification of developing ferret brain by magnetic resonance imaging. J Anat 2007; 210(1): 66-77.

[19] Zilles K, Armstrong E, Moser KH, Schleicher A, Stephan H. Gyrification in the cerebral cortex of primates. Brain Behav Evol 1989; 34(3): 143-50.

[20] Liu F, Garland M, Duan Y, et al. Study of the development of fetal baboon brain using magnetic resonance imaging at 3 Tesla. Neuroimage 2008; 40(1): 148-59.

[21] Hutsler JJ, Lee DG, Porter KK. Comparative analysis of cortical layering and supragranular layer enlargement in rodent carnivore and primate species. Brain Res 2005; 1052(1): 71-81.

[22] Kochunov P, Duff DM. Development of structural MR brain imaging protocols to study genetics and maturation. Methods 2009; 50(3): 136-46.

[23] VandeBerg JL, Williams-Blangero S, Tardif S. The Baboon in Biomedical Research. New York: Springer 2009.

[24] Rogers J, Kochunov P, Lancaster J, et al. Heritability of brain volume, surface area and shape: An MRI study in an extended pedigree of baboons. Hum Brain Mapp 2007; 28(6): 576-83.

[25] Kochunov P, Castro C, Davis D, et al. Mapping primary gyrogenesis during fetal development in primate brains: highresolution in utero structural MRI study of fetal brain development in pregnant baboons. Front Neurosci 2010; 4: 20.

[26] Kochunov P, Lancaster JL, Glahn DC, et al. Retrospective motion correction protocol for high-resolution anatomical MRI. Hum Brain Mapp 2006; 27(12): 957-62.

[27] Mugler JP, Brookeman JR. Three-dimensional magnetizationprepared rapid gradient-echo imaging (3D MP RAGE). Magn Reson Med 1990; 15(1): 152-7.

[28] Deichmann R, Good C, Josephs O, Ashburner J, Turner R. Optimization of 3-D MP-RAGE sequences for structural brain imaging. Neuroimage 2000; 12(1): 112-27. 
[29] Kochunov P, Lancaster J, Glahn DC, et al. A retrospective motion correction protocol for high-resolution anatomical MRI. Hum Brain Mapp 2006; 27(12): 957-62.

[30] Biegon A, Eberling JL, Richardson BC, et al. Human corpus callosum in aging and Alzheimer's disease: A magnetic resonance imaging study. Neurobiol Aging 1994; 15(4): 393-7.

[31] Sanchez MM, Hearn EF, Do D, Rilling JK, Herndon JG. Differential rearing affects corpus callosum size and cognitive function of rhesus monkeys. Brain Res 1988; 812: 38-49.

[32] Phillips KA, Sherwood CC, Lilak AL. Corpus callosum morphology in capuchin monkeys is influenced by sex and handendess. PLosONE 2007; 2(8): 1-7.

[33] Pierre PJ, Hopkins WD, Taglialatela JP, Lees CJ, Bennett AJ. Agerelated neuroanatomical differences from the juvenile period to adulthood in mother-reared macaques (Macaca radiata). Brain Res 2008; 126: 56-60.

[34] Aboitiz F, Scheibel AB, Fisher RS, Zaidel E. FIber composition of the human corpus callosum. Brain Res 1992; 598(1-2): 143-53.

[35] Alexander AL, Lee JE, Lazar M, et al. Diffusion tensor imaging of the corpus callosum in autism. Neuroimage 2007; 34: 61-73.

[36] Hofer S, Frahn J. Topography of the human corpus callosum revisited - Comprehensive fiber tractography using diffusion tensor magnetic resonance imaging. Neuroimage 2006; 32: 989-94.

[37] Allen LS, Richey MF, Chai YM, Gorskiu RA. Sex differences in the corpus callosum of the living human being. J Neurosci 1991; 11: 933-42.

[38] Hasan KM, Kamali A, Iftikhar A, et al. Diffusion tensor tractography quantification of the human corpus callosum fiber pathways across the lifespan. Brain Res 2009; 1249: 91-100.

[39] Moffat SD, Hampson E, Wickett JC, Vernon PA, Lee DH. Testosterone is correlated with regional morphology of the human corpus callosum. Brain Res 1997; 767: 297-304.

[40] Phillips KA, Sherwood CC. Cortical development in brown capuchin monkeys: A structural MRI study. Neuroimage 2008; 43: 657-64.

[41] Hasan KM, Kamali A, Kramer LA, Papnicolaou AC, Fletcher JM, Ewing-Cobbs L. Diffusion tensor quantification of the human midsagittal corpus callosum subdivisions across the lifespan. Brain Res 2008; 1227: 52-67.

[42] McLaughlin NC, Paul RH, Grieve SM, et al. Diffusion tensor imaging of the corpus callosum: A cross-sectional study across the lifespan. Int J Dev Neurosci 2007; 25: 215-21.
[43] Pujol J, Vendrell P, Junque C, Marti-Vilalta JL, Capdevila A. When does human brain development end? Evidence of corpus callosum growth up to adulthood. Ann Neurol 1993; 34: 71-5.

[44] Rauch RA, Jinkins JR. Analysis of cross-sectional area measurements of the corpus callosum adjusted for brain size in male and female subjects from childhood to adulthood. Behav Brain Res 1994; 64: 65-78.

[45] LaMantia AS, Rakic P. Axon overproduction and elimination in the corpus callosum of the developing rhesus monkey. J Neurosci 1990; 10: 2156-75.

[46] Hopkins WD, Phillips KA. Cross-sectional analysis of the association between age and corpus callosum size in chimpanzees (Pan troglodytes). Dev Psychobiol 2010; 52(2): 133-41.

[47] Giedd JN, Rumsey JM, Castellanos FX, et al. A quantitative MRI study of the corpus callosum in children and adolescents. Brain Res Dev 1996; 91: 274-80.

[48] Giedd JN, Blumenthal J, Jeffries NO, et al. Development of the human corpus callosum during childhood and adolescence: a longituginal MRI study. Prog Neuropsychopharmacol Biol Psychiatry 1999; 23(4): 571-88.

[49] Rajapakse JC, Giedd JN, Rumsey JM, Vaituzis AC, Hamburger SD, Rapoport JL. Regional MRI measurements of the corpus callosum: a methodological and developmental study. Brain Dev 1996; 18(5): 379-88.

[50] Lenroot RK, Gogtay N, Greenstein DK, et al. Sexual dimorphism of brain developmental trajectories during childhood and adolescence. Neuroimage 2007; 36: 1065-73.

[51] Luders E, Thompson PM, Toga AW. The development of the corpus callosum in the healthy human brain. J Neurosci 2010; 30(33): 10985-90.

[52] Galaburda A, Rosen GD, Sherman GF. Individual variability in cortical organization: Its relationship to brain laterality and implications to function. Neuropsychologia 1990; 28: 529-46.

[53] Luo L, O'Leary DD. Axon retraction and degeneration in development and disease. Annu Rev Neurosci 2005; 28: 127-56.

[54] Barazany D, Basser PJ, Assaf Y. In vivo measurement of axon diameter distribution in the corpus callosum of rat brain. Brain 2009; 132: 1210-20.

[55] Stewart CB, Disotell TR. Primate evolution - in and out of Africa. Curr Biol 1998; 8(16): R582-8.

[56] Levitt P. Structural and functional maturation of the developing primate brain. J Pediatr 2003; 143(4 Suppl): S35-45.

[57] Van Essen DC, Dierker DL. Surface-based and probabilistic atlases of primate cerebral cortex. Neuron 2007; 56(2): 209-25.

(c) Phillips and Kochunov; Licensee Bentham Open.

This is an open access article licensed under the terms of the Creative Commons Attribution Non-Commercial License (http://creativecommons.org/licenses/by-nc/3.0/) which permits unrestricted, non-commercial use, distribution and reproduction in any medium, provided the work is properly cited. 